\title{
TRANSIENT VISCOUS FLOW IN AN ANNULUS
}

\author{
A.A. KOLYSHKIN and I. VOLODKO \\ Department of Engineering Mathematics, Riga Technical University \\ Meza 1/4, Riga, LV 1048 Latvia \\ E-mail: koliskins@rbi.lv inta@egle.cs.rtu.lv
}

Received September 30, 2002; revised November 11, 2002

\begin{abstract}
The method of matched asymptotic expansions is used in the present paper to derive an approximate solution for transient flow of a viscous incompressible fluid in an annulus. The transient is caused by a sudden reduction of flow rate to zero. The laminar flow before deceleration can be either steady or unsteady but unidirectional. The solution is valid for short time intervals after sudden deceleration.
\end{abstract}

Key words: incompressible fluid flow, asymptotic expansion.

\section{INTRODUCTION}

Unsteady flows of a viscous incompressible fluid are important in many engineering problems. A sudden change of the flow rate can occur in some hydraulic problems (waterhammer) or in the flow of blood in the aorta. As a result, the velocity and pressure distribution change considerably during short time interval and can cause a severe damage to pipelines and other hydraulic devices.

There are not too many known analytical solutions for unsteady viscous flow in pipes and channels. Historically the first one was the problem solved by Gromeka [3]. He considered an impulsive start of the flow in a circular cylinder due to instantaneous constant pressure gradient. A laminar decay of a fully developed flow in pipes and channels after a sudden reduction of the flow rate to zero is studied by Weinbaum and Parker [7]. Their solution is found by means of the Pohlhausen method used in boundary layer theory. Das and Arakeri [1] obtained some analytical solutions for unsteady laminar flows in pipes and channels for the case where the motion is generated by a 
piston. The solutions in [1] correspond to the case where the velocity of the piston is either constant over some time interval or varies linearly with time.

The solutions in $[1 ; 3]$ are expressed in terms of infinite series and can be used in practice only if the parameter $\beta=\nu t^{*} / R_{0}^{2}$ is not too small, where $\nu$ is the viscosity of the fluid, $t^{*}$ is time and $R_{0}$ is the radius of the pipe. However, there are cases where the parameter $\beta$ can be very small. For example, in waterhammer analysis the time scale is of order a few seconds, therefore $\beta$ can be in the range $10^{-6}$ to $10^{-4}$. The infinite series in $[1 ; 3]$ are practically useless in such a case since too many terms in the series should be taken into account in order to obtain the velocity distribution.

For small values of $\beta$ the method of matched asymptotic expansions can give relatively simple approximate solutions which can be successfully used in practice. Examples of such solutions for the case of a plane channel and a pipe are given in $[2 ; 6]$. It is also shown in [2] that for short time intervals the approximate solution for unsteady flow in a pipe after a sudden blockage agrees very well with exact analytical solution. Asymptotic solution for unsteady viscous flow in an annulus after a sudden reduction of the flow rate to zero is obtained in the present paper. The solution is found by matching the solutions in the core and two boundary layers near the walls of the annulus.

\section{ASYMPTOTIC SOLUTION}

Consider an infinitely long annulus of the inner and outer radii $R_{1}$ and $R_{2}$, respectively, filled with a viscous incompressible fluid. At time $t_{*}=0$ the flow is instantaneously decelerated so that the total fluid flux through the cross section of the annulus is zero. We introduce a system of cylindrical polar coordinates $\left(r_{*}, \theta, z_{*}\right)$ with the origin at the common axis of the cylinders. It is assumed that the flow before and after deceleration is unidirectional, that is, the velocity vector has only one nonzero (longitudinal) component which is a function of $r_{*}$ and $t_{*}$ only. The results of Weinbaum and Parker [7] show that this is true at a distance of order channel width (in the case of a plane channel) from the point where the flow is blocked. In this case the system of Navier-Stokes equations reduces to the following equation

$$
\frac{\partial u_{*}}{\partial t_{*}}=-\frac{1}{\rho} \frac{\partial p_{*}}{\partial z_{*}}+\nu\left(\frac{\partial^{2} u_{*}}{\partial r_{*}^{2}}+\frac{1}{r_{*}} \frac{\partial u_{*}}{\partial r_{*}}\right) .
$$

The following dimensionless variables are used in sequel: $r=r_{*} / R_{2}, t=t_{*} / T$, $u=u_{*} / U_{*}$ and $p=p_{*} T /\left(\rho U_{*} R_{2}\right)$, where $T$ is some characteristic time and $U_{*}$ is some characteristic velocity. Then the dimensionless form of equation $(2.1)$ is

$$
\frac{\partial u}{\partial t}=\varphi(t)+\varepsilon\left(\frac{\partial^{2} u}{\partial r^{2}}+\frac{1}{r} \frac{\partial u}{\partial r}\right)
$$


where we used the notations $\varepsilon=\frac{\nu T}{R_{2}^{2}}$ and $\varphi(t)=-\frac{\partial p}{\partial z}$. It follows from equation (2.1) that if $u_{*}$ is a function of $r_{*}$ and $t_{*}$ only then the pressure gradient with respect to $z_{*}$ is a function of time only. The parameter $\varepsilon$ is the ratio of the wave time scale, $T$, and the diffusion time scale, $R_{2}^{2} / \nu$. In some applications the value of $\varepsilon$ can be very small. For example, in waterhammer experiments of Holomboe and Roleau [4] the value of $\varepsilon=0.0066$ for the laminar case. The boundary conditions are

$$
\left.u\right|_{r=R}=0,\left.\quad u\right|_{r=1}=0
$$

where $R=R_{1} / R_{2}$ is the radius ratio of the cylinders. The initial condition is given by

$$
\left.u\right|_{t=0}=g(r),
$$

where $g(r)$ is the function which represents the velocity distribution before deceleration. The non-slip boundary conditions on the walls $r=1$ and $r=R$ are assumed to be satisfied for $g(r)$, that is, $g(1)=0$ and $g(R)=0$ so that the boundary conditions (2.3) and initial conditions (2.4) are consistent. Note that the flow before deceleration can be either steady or unsteady, but unidirectional.

Finally, the total fluid flux through the cross section of the annulus is zero for all $t>0$, that is,

$$
\frac{2}{1-R^{2}} \int_{R}^{1} r u d r=0 .
$$

Condition (2.5) is an idealized condition and corresponds to the case of instantaneous closure of the annulus. In more realistic cases the reduction of fluid flux to zero occurs during short but finite time interval. Applying the Laplace transform to $(2.2)-(2.5)$ we obtain

$$
\begin{gathered}
s \bar{u}-g(r)=\bar{\varphi}+\varepsilon\left(\frac{d^{2} \bar{u}}{d r^{2}}+\frac{1}{r} \frac{d \bar{u}}{d r}\right), \\
\left.\bar{u}\right|_{r=R}=0,\left.\quad \bar{u}\right|_{r=1}=0, \\
\frac{2}{1-R^{2}} \int_{R}^{1} r \bar{u} d r=0,
\end{gathered}
$$

where $s$ is the parameter of the Laplace transform, $\bar{u}$ and $\bar{\varphi}$ are the Laplace transforms of the functions $u$ and $\varphi$, respectively.

The method of matched asymptotic expansions is used in the present paper to construct asymptotic solution for $(2.6)-(2.8)$ valid for short times.

The outer expansions for the functions $\bar{u}(r, s)$ and $\bar{\varphi}(s)$ are sought in the form

$$
\begin{aligned}
\bar{u}(r, s, \varepsilon) & =\bar{u}_{0}(r, s)+\sqrt{\varepsilon} \bar{u}_{1}(r, s)+\varepsilon \bar{u}_{2}(r, s)+\ldots \\
\bar{\varphi}(s, \varepsilon) & =\bar{\varphi}_{0}(s)+\sqrt{\varepsilon} \bar{\varphi}_{1}(s)+\varepsilon \bar{\varphi}_{2}(s)+\ldots
\end{aligned}
$$


Substituting (2.9), (2.10) into (2.6), (2.8) and collecting the terms that do not contain $\varepsilon$, we obtain

$$
\begin{gathered}
s \bar{u}_{0}-g(r)=\bar{\varphi}_{0}, \\
\frac{2}{1-R^{2}} \int_{R}^{1} r \bar{u}_{0} d r=0 .
\end{gathered}
$$

Solving (2.11) and (2.12) yields

$$
\bar{\varphi}_{0}=-G, \quad \bar{u}_{0}=\frac{g(r)-G}{s},
$$

where $G$ is the average velocity of the flow before deceleration:

$$
G=\frac{2}{1-R^{2}} \int_{R}^{1} r g(r) d r .
$$

The inner expansion near the wall $r=R$ is sought in the form

$$
\bar{u}(r, s, \varepsilon)=\bar{U}_{0}(\xi, s)+\sqrt{\varepsilon} \bar{U}_{1}(\xi, s)+\varepsilon \bar{U}_{2}(\xi, s)+\ldots,
$$

where $\xi$ is the boundary layer variable defined by

$$
\xi=\frac{r-R}{\sqrt{\varepsilon}} .
$$

Substituting (2.14) into (2.6), (2.7) and collecting the terms that do not contain $\varepsilon$, we obtain

$$
\frac{d^{2} \bar{U}_{0}}{d \xi^{2}}-s \bar{U}_{0}=G
$$

and

$$
\bar{U}_{0}(\xi=0, s)=0 .
$$

The function $U_{0}(\xi, t)$ describes the development of the boundary layer near the wall to order unity. Since diffusion was absent at $t=0$, in order to derive (2.15) zero initial condition for $U_{0}$ is used. General solution of equation (2.15) has the form

$$
\bar{U}_{0}(\xi ; s)=C_{1} e^{\sqrt{s} \xi}+C_{2} e^{-\sqrt{s} \xi}-\frac{G}{s},
$$

where $C_{1}$ and $C_{2}$ are arbitrary constants. Using the matching condition

$$
\lim _{\xi \rightarrow \infty} \bar{U}_{0}=\lim _{r \rightarrow R} \bar{u}_{0}
$$


we obtain that $C_{1}=0$. The value of the constant $C_{2}$ can be found from (2.16). Thus the boundary correction near the wall $r=R$ to order unity is

$$
\bar{U}_{0}(\xi, s)=\frac{G}{s}\left(e^{-\sqrt{s} \xi}-1\right) .
$$

The inner expansion near the wall $r=1$ is sought in the form

$$
\bar{u}(r, s, \varepsilon)=\bar{V}_{0}(\eta, s)+\sqrt{\varepsilon} \bar{V}_{1}(\eta, s)+\varepsilon \bar{V}_{2}(\eta, s)+\ldots,
$$

where $\eta$ is the boundary layer variable defined by

$$
\eta=\frac{1-r}{\sqrt{\varepsilon}}
$$

The function $\bar{V}_{0}$ satisfies the following equation

$$
\frac{d^{2} \bar{V}_{0}}{d \eta^{2}}-s \bar{V}_{0}=G
$$

and the boundary condition

$$
\bar{V}_{0}(\eta=0, s)=0 .
$$

The solution to $(2.17),(2.18)$ that satisfies the matching condition

$$
\lim _{\eta \rightarrow \infty} \bar{V}_{0}=\lim _{r \rightarrow 1} \bar{u}_{0}
$$

can be written as follows

$$
\bar{V}_{0}(\eta, s)=\frac{G}{s}\left(e^{-\sqrt{s} \eta}-1\right) .
$$

In order to construct a uniformly valid $(R \leq r \leq 1)$ approximation to order unity one should add functions $\bar{u}_{0}$ and $\bar{U}_{0}$ and then subtract their common part (see, for example, Kevorkian and Cole [5]):

$$
\bar{u}(r, s, \varepsilon)=\frac{g(r)}{s}+\frac{G}{s}\left\{\exp \left[-\sqrt{s} \frac{(r-R)}{\sqrt{\varepsilon}}\right]+\exp \left[-\sqrt{s} \frac{(1-r)}{\sqrt{\varepsilon}}\right]-1\right\} .
$$

It is clear that the function (2.19) does not satisfy (to order $O(\sqrt{\varepsilon})$ ) the condition of zero flux through the cross section of the annulus. Substituting (2.19) into (2.8) yields

$$
\frac{2}{1-R^{2}} \int_{R}^{1} r \bar{u}(r, s, \varepsilon) d r=\frac{2}{1-R} \frac{G}{s \sqrt{s}} \sqrt{\varepsilon}+O(\varepsilon) .
$$


On the other hand, substituting (2.9) into (2.8) we obtain

$$
\frac{2}{1-R^{2}} \int_{R}^{1}\left[\bar{u}_{0}(r, s)+\sqrt{\varepsilon} \bar{u}_{1}(r, s)+\ldots\right] r d r=0
$$

Comparing (2.20) and (2.21) and using (2.13) yield

$$
\frac{2}{1-R^{2}} \int_{R}^{1} r \bar{u}_{1}(r, s) d r=-\frac{2 G}{(1-R) s \sqrt{s}}
$$

Substituting (2.9) and (2.10) into (2.6) and collecting terms of order $O(\sqrt{\varepsilon})$ we obtain

$$
s \bar{u}_{1}=\bar{\varphi}_{1}
$$

It follows from (2.22) and (2.23) that

$$
\bar{u}_{1}(r, s)=-\frac{2 G}{(1-R) s \sqrt{s}}, \quad \bar{\varphi}_{1}(s)=-\frac{2 G}{(1-R) \sqrt{s}} .
$$

The equation for the boundary correction $\bar{U}_{1}(\xi, s)$ near the wall $r=R$ is obtained by substituting (2.14) into (2.6) and (2.7) and collecting terms of order $O(\sqrt{\varepsilon})$ :

$$
\frac{d^{2} \bar{U}_{1}}{d \xi^{2}}-s \bar{U}_{1}=\frac{2 G}{(1-R) \sqrt{s}}+\frac{G}{R \sqrt{s}} \exp (-\sqrt{s} \xi)
$$

The boundary condition is

$$
\bar{U}_{1}(\xi=0, s)=0
$$

Using the matching condition

$$
\lim _{\xi \rightarrow \infty} \bar{U}_{1}(\xi, s)=\lim _{r \rightarrow R} \bar{u}_{1}(r, s)
$$

and solving (2.24), (2.25) we obtain

$$
\bar{U}_{1}(\xi, s)=\frac{2 G}{(1-R) s \sqrt{s}}[\exp (-\sqrt{s} \xi)-1]-\frac{G}{2 R s} \xi \exp (-\sqrt{s} \xi)
$$

The boundary correction $\bar{V}_{1}(\eta, s)$ can be found in a similar way and has the form

$$
\bar{V}_{1}(\eta, s)=\frac{2 G}{(1-R) s \sqrt{s}}[\exp (-\sqrt{s} \eta)-1]-\frac{G}{2 s} \eta \exp (-\sqrt{s} \eta)
$$


The Laplace transform of the solution up to $O(\varepsilon)$ is

$$
\begin{aligned}
\bar{u}(r, s)= & \frac{g(r)}{s}+\frac{G}{s}\left\{\frac{(3 R-r)}{2} \exp \left[-\frac{\sqrt{s}}{\sqrt{\varepsilon}}(r-R)\right]\right. \\
& \left.+\frac{(1+r)}{2} \exp \left[-\frac{\sqrt{s}}{\sqrt{\varepsilon}}(1-r)\right]-1\right\} \\
& +\sqrt{\varepsilon} \frac{2 G}{(1-R) s \sqrt{s}}\left\{\exp \left[-\frac{\sqrt{s}}{\sqrt{\varepsilon}}(r-R)\right]\right. \\
& \left.+\exp \left[-\frac{\sqrt{s}}{\sqrt{\varepsilon}}(1-r)\right]-1\right\}+O(\varepsilon) .
\end{aligned}
$$

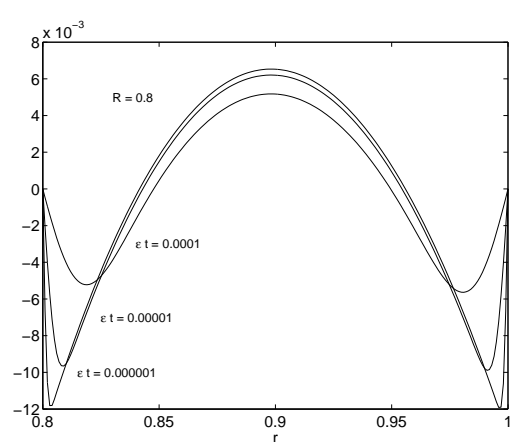

Figure 1.

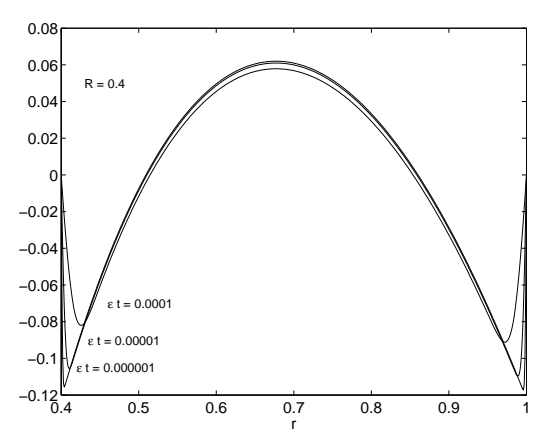

Figure 2.

Applying the inverse Laplace transform to (2.26) we obtain the solution to $(2.2)-(2.5)$ up to $O(\varepsilon)$ in the form (other terms of the asymptotic expansion can be constructed in a similar way):

$$
\begin{aligned}
u(r, t)= & g(r)-G+\frac{G}{2 R(1-R)}\left(R^{2}+3 R-3 R r-r\right) \operatorname{erfc} \frac{r-R}{2 \sqrt{\varepsilon t}} \\
& +\frac{G}{2(1-R)}(-3-R+5 r-R r) \operatorname{erfc} \frac{1-r}{2 \sqrt{\varepsilon t}} \\
& +\frac{4 G}{1-R} \sqrt{\frac{\varepsilon t}{\pi}}\left\{\exp \left[-\frac{(r-R)^{2}}{4 \varepsilon t}\right]+\exp \left[-\frac{(1-r)^{2}}{4 \varepsilon t}\right]-1\right\} \\
& +O(\varepsilon) .
\end{aligned}
$$

Figures 1 and 2 show the velocity distribution given by (2.27) where

$$
g(r)=1-r^{2}-\frac{\left(1-R^{2}\right)}{\ln R} \ln r
$$


and

$$
G=\frac{1+R^{2}}{2}+\frac{1-R^{2}}{2 \ln R}
$$

The function (2.28) corresponds to the case of fully developed Poiseuille flow in the annulus before sudden blockage. Both graphs indicate that the velocity profile rapidly changes in the boundary layers near the walls while the core region is characterized by relatively slow variation of the velocity.

\section{REFERENCES}

[1] D. Das and J. H. Arakeri. Transition of unsteady velocity profiles with reverse flow. $J$. Fluid Mech., 374, 251 - 283, 1998.

[2] M. Ghidaoui and A. A. Kolyshkin. On the theory of unsteady flows in a pipe. J. Fluid Mech., 2002. (submitted)

[3] I. S. Gromeka. On the theory of fluid motion in narrow cylindrical pipes. Kazan University Research Notes, 1882. (In Russian)

[4] E. L. Holomboe and W. T. Roleau. The effect of viscous shear on transients in liquid lines. J. Basic Eng., 89, $174-180,1967$.

[5] J. Kevorkian and J. D. Cole. Multiple scale and singular peturbation methods. Springer, New York, 1996.

[6] A. A. Kolyshkin and R. Vaillancourt. Asymptotic solution for unsteady viscous flow in a plane channel. Latvian J. Physical an Technical Sciences, 3, 12-19, 2001.

[7] S. Weinbaum and K. H. Parker. The laminar decay of suddenly blocked channel and pipe flows. J. Fluid Mech., 69, $729-752,1975$.

\section{Pereinamasis klampusis tekẻjimas žiede}

\section{A. Kolyshkin, I. Volodko}

Išnagrinètas klampiojo nespūdaus skysčio vienas atvejis, kai procesas modeliuojamas antrosios eilès dierencialine lygtimi su mažu parametru. Sukonstruotas uždavinio su pradine, kraštinèmis bei su nelokaliaja sąlygomis formalusis asimptotinis sprendinys. 